DOI 10.4171/JEMS/236

Fernando Mário de Oliveira Filho · Frank Vallentin

\title{
Fourier analysis, linear programming, and densities of distance avoiding sets in $\mathbb{R}^{n}$
}

Received August 20, 2008 and in revised form November 19, 2008

\begin{abstract}
We derive new upper bounds for the densities of measurable sets in $\mathbb{R}^{n}$ which avoid a finite set of prescribed distances. The new bounds come from the solution of a linear programming problem. We apply this method to obtain new upper bounds for measurable sets which avoid the unit distance in dimensions $2, \ldots, 24$. This gives new lower bounds for the measurable chromatic number in dimensions $3, \ldots, 24$. We apply it to get a short proof of a variant of a recent result of Bukh which in turn generalizes theorems of Furstenberg, Katznelson, Weiss, Bourgain and Falconer about sets avoiding many distances.
\end{abstract}

Keywords. Measurable chromatic number, linear programming, autocorrelation function

\section{Introduction}

Let $d_{1}, \ldots, d_{N}$ be positive real numbers. We say that a subset $A$ of the $n$-dimensional Euclidean space $\mathbb{R}^{n}$ avoids the distances $d_{1}, \ldots, d_{N}$ if the distance between any two points in $A$ is never $d_{1}, \ldots, d_{N}$. We define the upper density of a Lebesgue measurable set $A \subseteq \mathbb{R}^{n}$ as

$$
\bar{\delta}(A)=\limsup _{T \rightarrow \infty} \frac{\operatorname{vol}\left(A \cap[-T, T]^{n}\right)}{\operatorname{vol}[-T, T]^{n}} .
$$

In this expression $[-T, T]^{n}$ denotes the regular cube in $\mathbb{R}^{n}$ with side $2 T$ centered at the origin. We denote the extreme density which a measurable set in $\mathbb{R}^{n}$ that avoids the distances $d_{1}, \ldots, d_{N}$ can have by

$m_{d_{1}, \ldots, d_{N}}\left(\mathbb{R}^{n}\right)=\sup \left\{\bar{\delta}(A): A \subseteq \mathbb{R}^{n}\right.$ is measurable and avoids the distances $\left.d_{1}, \ldots, d_{N}\right\}$.

In this paper we derive upper bounds for this extreme density from the solution of a linear programming problem.

To formulate our main theorem we consider the function $\Omega_{n}$ given by

$$
\Omega_{n}(t)=\Gamma\left(\frac{n}{2}\right)\left(\frac{2}{t}\right)^{\frac{1}{2}(n-2)} J_{\frac{1}{2}(n-2)}(t) \quad \text { for } t>0, \quad \Omega_{n}(0)=1,
$$

F. M. de Oliveira Filho: Department of Econometrics and OR, Tilburg University, 5000 LE Tilburg, The Netherlands; e-mail: F.M.deOliveiraFilho@uvt.nl

F. Vallentin: Delft Institute of Applied Mathematics, Technical University of Delft, P.O. Box 5031, 2600 GA Delft, The Netherlands; e-mail: f.vallentin@tudelft.nl

Mathematics Subject Classification (2010): 42B05, 52C10, 52C17, 90C05 


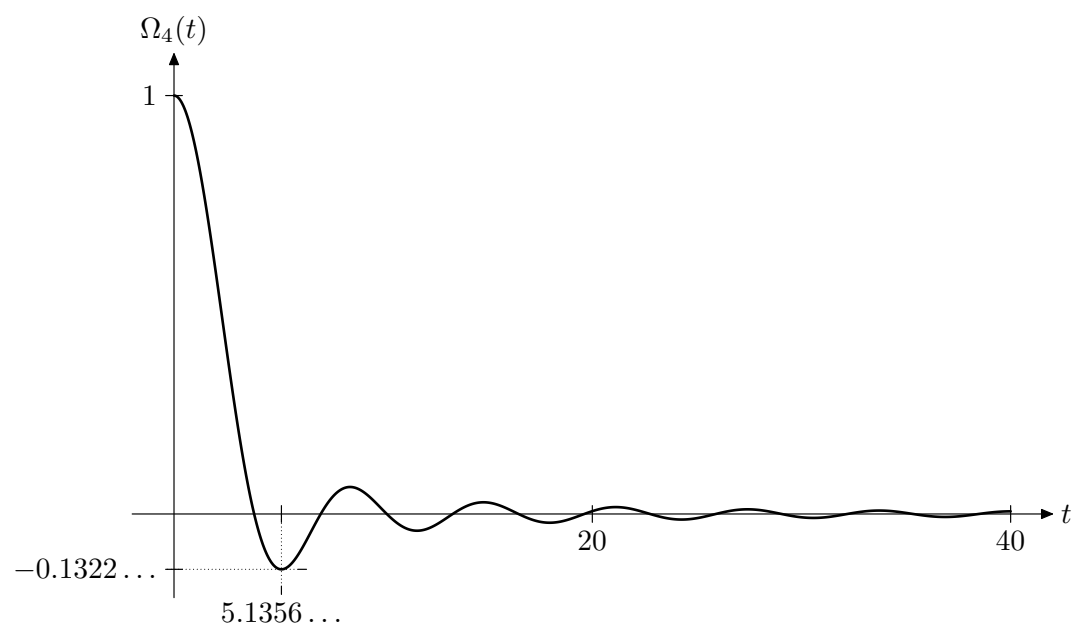

Fig. 1.1. Graph of the function $\Omega_{4}(t)=\frac{2}{t} J_{1}(t)$

where $J_{\frac{1}{2}(n-2)}$ is the Bessel function of the first kind with parameter $(n-2) / 2$. To fix ideas we plotted the graph of the function $\Omega_{4}$ in Figure 1.1

Theorem 1.1. Let $d_{1}, \ldots, d_{N}$ be positive real numbers. Let $A \subseteq \mathbb{R}^{n}$ be a measurable set which avoids the distances $d_{1}, \ldots, d_{N}$. Suppose there are real numbers $z_{0}, z_{1}, \ldots, z_{N}$ which sum up to at least one and which satisfy

$$
z_{0}+z_{1} \Omega_{n}\left(t d_{1}\right)+\cdots+z_{N} \Omega_{n}\left(t d_{N}\right) \geq 0
$$

for all $t \geq 0$. Then the upper density of $A$ is at most $z_{0}$.

In Section 2 we provide a proof where we make essential use of basic harmonic analysis, which we briefly recall. In the sections that follow we apply the main theorem in a variety of situations: for sets avoiding one distance, sets avoiding two distances, and sets avoiding many distances. For the history of these Euclidean distance problems we refer to the surveys by Székely [19] and Székely and Wormald [20] and the references therein.

Sets avoiding one distance have been studied by combinatorialists because of their relation to the measurable chromatic number of the Euclidean space. This is the minimum number of colors one needs to color all points in $\mathbb{R}^{n}$ so that any two points at distance 1 receive different colors and so that points receiving the same color form Lebesgue measurable sets; it will be denoted by $\chi_{\mathrm{m}}\left(\mathbb{R}^{n}\right)$. Since every color class of a coloring provides a measurable set which avoids the distance 1 , we have the inequality

$$
m_{1}\left(\mathbb{R}^{n}\right) \cdot \chi_{\mathrm{m}}\left(\mathbb{R}^{n}\right) \geq 1 \text {. }
$$

For the plane it is only known that $5 \leq \chi_{\mathrm{m}}\left(\mathbb{R}^{2}\right) \leq 7$, where the lower bound is due to Falconer [10] and the upper bound comes e.g. from a coloring one constructs using a tiling by regular hexagons with circumradius slightly less than 1. Erdős conjectured that 
$m_{1}\left(\mathbb{R}^{2}\right)<1 / 4$ so that 2 would yield an alternative proof of Falconer's result. So far the best known results on $m_{1}\left(\mathbb{R}^{2}\right)$ are the lower bound $m_{1}\left(\mathbb{R}^{2}\right) \geq 0.2293$ by Croft [7] and the upper bound $m_{1}\left(\mathbb{R}^{2}\right) \leq 12 / 43 \approx 0.2790$ by Székely [18]. In Section 3 we compute new upper bounds for $m_{1}\left(\mathbb{R}^{n}\right)$ for dimensions $n=2, \ldots, 24$ based on a strengthening of our main theorem by extra inequalities. These new upper bounds for $m_{1}\left(\mathbb{R}^{n}\right)$ imply by (2) new lower bounds for $\chi_{\mathrm{m}}\left(\mathbb{R}^{n}\right)$ in dimensions $3, \ldots, 24$.

If one considers sets which avoid more than one distance one can ask how $N$ distances can be chosen so that the extreme density becomes as small as possible: what is the value of $\inf \left\{m_{d_{1}, \ldots, d_{N}}\left(\mathbb{R}^{n}\right): d_{1}, \ldots, d_{N}>0\right\}$ for fixed $N$ ? For planar sets avoiding two distances Székely [18] showed that $\inf \left\{m_{d_{1}, d_{2}}\left(\mathbb{R}^{2}\right): d_{1}, d_{2}>0\right\} \leq m_{1, \sqrt{3}}\left(\mathbb{R}^{2}\right) \leq$ $2 / 11 \approx 0.181818$. In Section 4 we improve his result and show that $\inf \left\{m_{d_{1}, d_{2}}\left(\mathbb{R}^{2}\right)\right.$ : $\left.d_{1}, d_{2}>0\right\} \leq 0.0724046$.

Recently, Bukh [5], using harmonic analysis and ideas resembling Szémeredi's regularity lemma, showed that $\inf \left\{m_{d_{1}, \ldots, d_{N}}\left(\mathbb{R}^{n}\right): d_{1}, \ldots, d_{N}>0\right\}$ drops to zero exponentially in $N$ : he shows that there is a number $r$, strictly greater than 1 , which depends only on $N$ and $n$ so that if

$$
d_{2} / d_{1}>r, \quad d_{3} / d_{2}>r, \ldots, d_{N} / d_{N-1}>r
$$

then $m_{d_{1}, \ldots, d_{N}}\left(\mathbb{R}^{n}\right) \leq\left(m_{1}\left(\mathbb{R}^{n}\right)\right)^{N}$. This implies a theorem of Furstenberg, Katznelson, and Weiss [13] that for every subset $A$ in the plane which has positive upper density there is a constant $d$ so that $A$ does not avoid distances larger than $d$. Their original proof used tools from ergodic theory and measure theory. Alternative proofs have been proposed by Bourgain [4] using elementary harmonic analysis and by Falconer and Marstrand [12] using geometric measure theory. Bukh's result also implies that $m_{d_{1}, \ldots, d_{N}}\left(\mathbb{R}^{n}\right)$ becomes arbitrarily small if the distances $d_{1}, \ldots, d_{N}$ become sufficiently small. This is originally due to Bourgain [4] and Falconer [11]. In Section 5] we give a short proof of a variant of Bukh's result using our main theorem, where we replace $\left(m_{1}\left(\mathbb{R}^{n}\right)\right)^{N}$ by the weaker estimate $2^{-N}$. We could improve this considerably, but we cannot get $\left(m_{1}\left(\mathbb{R}^{n}\right)\right)^{N}$. Still our estimate is strong enough to give all the implications mentioned. Furthermore, our proof has the additional advantage that it easily provides quantitative estimates on the spacing $r$ between the distances.

The idea of linear programming bounds for packing problems of discrete point sets in compact metric spaces goes back to Delsarte [9] and it has been successfully applied to a variety of situations. Cohn and Elkies [6] were the first who were able to set up a linear programming bound for packing problems in noncompact spaces; by then no less than 30 years since Delsarte's fundamental contribution had gone by. Our main theorem can be viewed as a continuous analogue to their linear programming bound.

\section{Proof of the main theorem}

For the proof of our main theorem, elementary notions from harmonic analysis will be important. We recall these here. For details we refer to, e.g., the book by Katznelson [14]. 
A measurable, complex valued function $f: \mathbb{R}^{n} \rightarrow \mathbb{C}$ is called periodic if it is invariant under an $n$-dimensional discrete subgroup of $\mathbb{R}^{n}$ or, in other words, if there is a basis $b_{1}, \ldots, b_{n}$ of $\mathbb{R}^{n}$ so that for all $\alpha_{1}, \ldots, \alpha_{n} \in \mathbb{Z}$ we have $f\left(x+\sum_{i=1}^{n} \alpha_{i} b_{i}\right)=f(x)$. The set $L=\left\{\sum_{i=1}^{n} \alpha_{i} b_{i}: \alpha_{i} \in \mathbb{Z}\right\}$ is called the period lattice of $f$ and $L^{*}=\left\{u \in \mathbb{R}^{n}\right.$ : $x \cdot u \in \mathbb{Z}$ for all $x \in L\}$ is called the dual lattice of $L$.

The mean value of a periodic function $f$ is given by

$$
M(f)=\lim _{T \rightarrow \infty} \frac{1}{\operatorname{vol}[-T, T]^{n}} \int_{[-T, T]^{n}} f(x) d x .
$$

For two periodic functions $f$ and $g$ we write $\langle f, g\rangle=M(f \bar{g})$. We say that $f$ is squareintegrable if $\langle f, f\rangle<\infty$. By $\|f\|=\sqrt{\langle f, f\rangle}$ we denote its norm. If $f$ and $g$ are both square-integrable, then $\langle f, g\rangle$ exists. For $u \in \mathbb{R}^{n}$ we define the Fourier coefficient $\widehat{f}(u)=$ $\left\langle f, e^{i u \cdot x}\right\rangle$. Here, $x \cdot y$ denotes the standard inner product on $\mathbb{R}^{n}$.

Notice that the support of $\widehat{f}$ is a discrete set, namely it lies in the dual lattice of the period lattice of $f$, scaled by $2 \pi$. If we let $f_{y}(x)=f(y+x)$ for a vector $y \in \mathbb{R}^{n}$, then $\widehat{f}_{y}(u)=\widehat{f}(u) e^{i u \cdot y}$. For square-integrable, periodic functions $f$ and $g$ Parseval's formula

$$
\langle f, g\rangle=\sum_{u \in \mathbb{R}^{n}} \widehat{f}(u) \overline{\widehat{g}(u)}
$$

holds. The latter sum is meant to extend over the intersection of the supports of $\widehat{f}$ and $\widehat{g}$.

Proof of Theorem 1.1. Let $A$ be a measurable subset of $\mathbb{R}^{n}$ that avoids the distances $d_{1}, \ldots, d_{N}$. Denote by $1_{A}$ its characteristic function $1_{A}: \mathbb{R}^{n} \rightarrow\{0,1\}$. Without loss of generality we can assume that $1_{A}$ is a periodic function; in this case we say that $A$ is periodic.

Indeed, from any measurable set $A$ which avoids the distances $d_{1}, \ldots, d_{N}$ we can construct a periodic set which avoids the distances $d_{1}, \ldots, d_{N}$ and with upper density arbitrarily close to the one of $A$. To do this we intersect $A$ with a regular cube of side $2 T$ so that $\operatorname{vol}\left(A \cap[-T, T]^{n}\right) / \operatorname{vol}[-T, T]^{n}$ is close to the upper density $\bar{\delta}(A)$ and so that $\operatorname{vol}[-T+d, T-d]^{n} / \operatorname{vol}[-T, T]^{n}$, with $d=\max \left\{d_{1}, \ldots, d_{N}\right\}$, differs from 1 only negligibly. Then we construct a new periodic set by tiling $\mathbb{R}^{n}$ with copies of $A \cap[-T+d$, $T-d]^{n}$ centered at the points of the lattice $2 T \mathbb{Z}^{n}$. Notice that, for a periodic set $A$, one may replace the lim sup in the definition of $\bar{\delta}(A)$ by a simple limit.

By $A-y$ we denote the translation of the set $A$ by the vector $-y \in \mathbb{R}^{n}$, so that $1_{A-y}(x)=1_{A}(x+y)=\left(1_{A}\right)_{y}(x)$. The following two properties are crucial:

$$
\begin{aligned}
\left\langle 1_{A}, 1\right\rangle & =\bar{\delta}(A), \\
\left\langle 1_{A-y}, 1_{A}\right\rangle & =\bar{\delta}(A \cap(A-y)) \quad \text { for all } y \in \mathbb{R}^{n} .
\end{aligned}
$$

In particular, $\left\langle 1_{A}, 1_{A}\right\rangle=\bar{\delta}(A)$ and $\left\langle 1_{A-y}, 1_{A}\right\rangle=0$ for all vectors $y$ of Euclidean norm $d_{1}, \ldots, d_{N}$. Notice $\left\langle 1_{A}, 1\right\rangle=\widehat{1_{A}}(0)$. By applying Parseval's formula to $(4)$, we can express it in terms of the Fourier coefficients of $1_{A}$, thus obtaining

$$
\begin{aligned}
\widehat{1_{A}}(0) & =\bar{\delta}(A), \\
\sum_{u \in \mathbb{R}^{n}}\left|\widehat{1_{A}}(u)\right|^{2} e^{i u \cdot y} & =\bar{\delta}(A \cap(A-y)) \quad \text { for all } y \in \mathbb{R}^{n} .
\end{aligned}
$$


Now we consider the function

$$
\varphi(y)=\sum_{u \in \mathbb{R}^{n}}\left|\widehat{1_{A}}(u)\right|^{2} e^{i u \cdot y}=\bar{\delta}(A \cap(A-y)),
$$

which is called the autocorrelation function (or two-point correlation function) of $1_{A}$. By taking spherical averages we construct from it a radial function $f$ whose values only depend on the norm of the vectors. In other words, we set

$$
f(y)=\frac{1}{\omega_{n}} \int_{S^{n-1}} \varphi(\|y\| \xi) d \omega(\xi) .
$$

Here $\omega$ denotes the standard surface measure on the unit sphere $S^{n-1}=\left\{\xi \in \mathbb{R}^{n}\right.$ : $\xi \cdot \xi=1\}$ and $\omega_{n}=\omega\left(S^{n-1}\right)=\left(2 \pi^{n / 2}\right) / \Gamma(n / 2)$. Clearly, $f(0)=\bar{\delta}(A)$, and $f(y)=0$ whenever $\|y\| \in\left\{d_{1}, \ldots, d_{N}\right\}$. Because of the formula (cf. Schoenberg [17, (1.6)], see (1) for an explicit expression for $\Omega_{n}$ )

$$
\frac{1}{\omega_{n}} \int_{S^{n-1}} e^{i u \cdot \xi} d \omega(\xi)=\Omega_{n}(\|u\|)
$$

we can represent $f$ in the form

$$
f(y)=\sum_{t \geq 0} \alpha(t) \Omega_{n}(t\|y\|),
$$

where $\alpha(t)$ is the sum of $\left|\widehat{1_{A}}(u)\right|^{2}$ for vectors $u$ having norm $t$, so the $\alpha(t)$ 's are real and nonnegative. Furthermore, $\alpha(0)=\left|\widehat{1_{A}}(0)\right|^{2}=\bar{\delta}(A)^{2}$ and $\sum_{t \geq 0} \alpha(t)=f(0)=\bar{\delta}(A)$.

So the following linear program in the variables $\alpha(t)$ gives an upper bound for the upper density of any measurable set which avoids the distances $d_{1}, \ldots, d_{N}$ :

$$
\begin{aligned}
\sup \{\alpha(0): \alpha(t) \geq 0 \text { for all } t \geq 0, & \sum_{t \geq 0} \alpha(t)=1, \\
& \left.\sum_{t \geq 0} \alpha(t) \Omega_{n}\left(t d_{k}\right)=0 \text { for } k=1, \ldots, N\right\} .
\end{aligned}
$$

Above, all but a countable subset of the $\alpha(t)$ 's are zero. Note moreover that we used the normalization $\sum_{t \geq 0} \alpha(t)=1$. This linear program has infinitely many variables $\alpha(t)$ but only $N+1$ equality constraints. A dual of it is

$$
\begin{aligned}
\inf \left\{z_{0}: z_{0}+z_{1}+\cdots+\right. & z_{N} \geq 1, \\
& \left.z_{0}+z_{1} \Omega_{n}\left(t d_{1}\right)+\cdots+z_{N} \Omega_{n}\left(t d_{N}\right) \geq 0 \text { for all } t>0\right\},
\end{aligned}
$$

which has $N+1$ variables $z_{0}, z_{1}, \ldots, z_{N}$ and infinitely many constraints. As usual, weak duality holds between the linear programs (6) and (7): If $\alpha(t)$ satisfies the conditions in (6) and if $\left(z_{0}, z_{1}, \ldots, z_{N}\right)$ satisfies the conditions in (7), then

$$
\alpha(0) \leq \sum_{t \geq 0} \alpha(t)\left(z_{0}+z_{1} \Omega_{n}\left(t d_{1}\right)+\cdots+z_{N} \Omega_{n}\left(t d_{N}\right)\right)=z_{0},
$$

which finishes the proof of our main theorem. 


\section{Sets avoiding one distance}

It is notable that the linear programming bounds for the extreme density of sets avoiding exactly one distance allow for an analytic optimal solution. Since this problem is scaling invariant we can assume that we consider sets avoiding the unit distance $d_{1}=1$. Let $j_{\alpha, k}$ be the $k$-th positive zero of the Bessel function $J_{\alpha}$. It is known that the absolute minimum of the function $\Omega_{n}$ is attained at $j_{n / 2,1}$ (see Andrews, Askey, and Roy [1, (4.6.2)], and Watson [21, Chapter 15, §31]). So, the point $\left(z_{0}, z_{1}\right)$ which is determined by the equations

$$
\begin{aligned}
z_{0}+z_{1} & =1, \\
z_{0}+z_{1} \Omega_{n}\left(j_{n / 2,1}\right) & =0,
\end{aligned}
$$

provides the optimal solution for the linear program in Theorem 1.1 . Hence,

$$
z_{0}=\Omega_{n}\left(j_{n / 2,1}\right) /\left(\Omega_{n}\left(j_{n / 2,1}\right)-1\right) \geq m_{1}\left(\mathbb{R}^{n}\right),
$$

and this gives by (2) a lower bound for the measurable chromatic number, namely $\chi_{\mathrm{m}}\left(\mathbb{R}^{n}\right)$ $\geq 1-1 / \Omega_{n}\left(j_{n / 2,1}\right)$. It is interesting to notice that this lower bound coincides with the one provided by Bachoc et al. [2, Corollary 8.2], albeit with a shift of one dimension. This shift is due to the fact that Bachoc et al. [2] study the problem of sets avoiding one distance on the $(n-1)$-dimensional unit sphere $S^{n-1} \subseteq \mathbb{R}^{n}$, and the lower bound for the measurable chromatic number $\chi_{\mathrm{m}}\left(\mathbb{R}^{n}\right)$ was obtained by upper bounding the density of sets in the unit sphere which avoid the distance $d$ where $d$ goes to zero. So, we see now that this limit process gives a lower bound for the measurable chromatic number of $\mathbb{R}^{n-1}$ and not only for $\mathbb{R}^{n}$.

\subsection{Adding extra inequalities}

It is possible to strengthen the main theorem and the resulting bound $(8)$ by introducing extra inequalities. Consider a regular simplex in $\mathbb{R}^{n}$ with edge length 1 having vertices $v_{1}, \ldots, v_{n+1}$. A set $A \subseteq \mathbb{R}^{n}$ which avoids the unit distance can only contain one vertex of this regular simplex. So for the autocorrelation function $\varphi$ of $1_{A}$ defined in (5) we have

$$
\varphi\left(v_{1}\right)+\cdots+\varphi\left(v_{n+1}\right)=\bar{\delta}\left(A \cap\left(A-v_{1}\right)\right)+\cdots+\bar{\delta}\left(A \cap\left(A-v_{n+1}\right)\right) \leq \bar{\delta}(A)=\varphi(0) .
$$

Let $\mathrm{O}\left(\mathbb{R}^{n}\right)$ be the $n$-dimensional orthogonal group, that is, the set of all $n \times n$ real matrices $Z$ such that $Z^{t} Z=I$. Let $\mu$ denote the Haar measure over $\mathrm{O}\left(\mathbb{R}^{n}\right)$ normalized by $\mu\left(\mathrm{O}\left(\mathbb{R}^{n}\right)\right)=1$. Taking spherical averages of $\varphi$ is the same as symmetrizing $\varphi$ with respect to the orthogonal group, i.e., for all $y \in \mathbb{R}^{n}$,

$$
f(y)=\frac{1}{\omega_{n}} \int_{S^{n-1}} \varphi(\|y\| \xi) d \omega(\xi)=\int_{\mathrm{O}\left(\mathbb{R}^{n}\right)} \varphi(Z y) d \mu(Z)
$$

(this follows, e.g., from Theorem 3.7 in the book by Mattila [15]). 
Let $f$ be, as above, the radial function obtained by the symmetrization of $\varphi$. For a nonnegative real number $t$, we write $f(t)$ for the common value of $f$ for vectors of length $t$. Then, by symmetrizing both sides of (9) with respect to the orthogonal group, and since distances are preserved by the action of $\mathrm{O}\left(\mathbb{R}^{n}\right)$, we conclude that the inequality

$$
f\left(\left\|v_{1}\right\|\right)+\cdots+f\left(\left\|v_{n+1}\right\|\right) \leq 1
$$

can be used to strengthen our original linear program. Here, observe that we already took into account the normalization $f(0)=1$, introduced in 6 .

If we center a regular simplex at the origin, the above inequality specializes to

$$
(n+1) f(\sqrt{1 / 2-1 /(2 n+2)}) \leq 1,
$$

which gives the following strengthening of the dual formulation (7):

$$
\begin{aligned}
& \inf \left\{z_{0}+z_{c}: z_{c} \geq 0, z_{0}+z_{1}+z_{c}(n+1) \geq 1\right. \\
& \left.\quad z_{0}+z_{1} \Omega_{n}(t)+z_{c}(n+1) \Omega_{n}(t \sqrt{1 / 2-1 /(2 n+2)}) \geq 0 \text { for all } t \geq 0\right\} .
\end{aligned}
$$

In Table 3.1 we give the new upper bounds on $m_{1}\left(\mathbb{R}^{n}\right)$ we get for $n=4, \ldots, 24$ by solving the linear program on a computer (we discuss numerical issues at the end of this section); these are improvements over the values which Székely and Wormald give in [20]. This in turn gives new lower bounds for the measurable chromatic number for $n=4, \ldots, 24$.

However, in dimension 2 we only get an upper bound of 0.287119 . To improve Székely's bound of $12 / 43 \approx 0.279069$ in the plane, we replace the regular triangle centered at the origin by more triangles. We use the following three triples of squared norms $\left(\left\|v_{1}\right\|^{2},\left\|v_{2}\right\|^{2},\left\|v_{3}\right\|^{2}\right)$ for $(10):(2.4,2.4,0.360314),(3.1,3.1,6.524038)$ $(3.7,3.7,7.417141)$, where the last coordinate of $(a, b, c)$ is a root of $3\left(a^{2}+b^{2}+c^{2}+1\right)-$ $(a+b+c+1)^{2}$. This condition ensures that the determinant of the positive semidefinite Gram matrix

$$
\left(\begin{array}{ccc}
a & \frac{1}{2}(a+b-1) & \frac{1}{2}(a+c-1) \\
\frac{1}{2}(a+b-1) & b & \frac{1}{2}(b+c-1) \\
\frac{1}{2}(a+c-1) & \frac{1}{2}(b+c-1) & c
\end{array}\right)
$$

of the points $v_{1}, v_{2}, v_{3}$ of a corresponding regular simplex vanishes. Solving the corresponding linear program yields the new upper bound of 0.268412 . We found the three triples by considering all triples $(a, b, c)$ with $a, b=0.1 j$ with $j=0, \ldots, 40$.

In dimension 3 we use three quadruples $\left(\left\|v_{1}\right\|^{2},\left\|v_{2}\right\|^{2},\left\|v_{3}\right\|^{2},\left\|v_{4}\right\|^{2}\right)$ of squared norms for (10): $(0.3,0.4,0.4,0.417157),(1.9,1.9,1.9,0.189372),(2,2,2,0.225148)$, where the last coordinate of $(a, b, c, d)$ is a root of $3\left(a^{2}+b^{2}+c^{2}+d^{2}+1\right)-2(a b+$ $a c+a d+b c+b d+c d)-2(a+b+c+d)$. Solving the corresponding linear programming problem yields the new upper bound of 0.165609 . We found the three quadruples by considering all triples $(a, b, c, d)$ with $a, b, c=0.1 j$ with $j=0, \ldots, 40$. 


\begin{tabular}{r|r|c|c|c}
\hline$n$ & $\begin{array}{c}\text { Best upper bound } \\
\text { for } m_{1}\left(\mathbb{R}^{n}\right) \\
\text { previously known }\end{array}$ & $\begin{array}{c}\text { New upper } \\
\text { bound for } \\
m_{1}\left(\mathbb{R}^{n}\right)\end{array}$ & $\begin{array}{c}\text { Best lower bound } \\
\text { for } \chi_{m}\left(\mathbb{R}^{n}\right) \\
\text { previously known }\end{array}$ & $\begin{array}{c}\text { New lower } \\
\text { bound for } \\
\chi_{m}\left(\mathbb{R}^{n}\right)\end{array}$ \\
\hline 2 & $0.279069[18]$ & 0.268412 & $5[10]$ & \\
3 & $0.187500[20]$ & 0.165609 & $6[10]$ & 7 \\
4 & $0.128000[20]$ & 0.112937 & $8[20]$ & 9 \\
5 & $0.0953947[20]$ & 0.0752845 & $11[20]$ & 14 \\
6 & $0.0708129[20]$ & 0.0515709 & $15[20]$ & 20 \\
7 & $0.0531136[20]$ & 0.0361271 & $19[20]$ & 28 \\
8 & $0.0346096[20]$ & 0.0257971 & $30[20]$ & 39 \\
9 & $0.0288215[20]$ & 0.0187324 & $35[20]$ & 54 \\
10 & $0.0223483[20]$ & 0.0138079 & $48[2]$ & 73 \\
11 & $0.0178932[20]$ & 0.0103166 & $64[2]$ & 97 \\
12 & $0.0143759[20]$ & 0.00780322 & $85[2]$ & 129 \\
13 & $0.0120332[20]$ & 0.00596811 & $113[2]$ & 168 \\
14 & $0.00981770[20]$ & 0.00461051 & $147[2]$ & 217 \\
15 & $0.00841374[20]$ & 0.00359372 & $191[2]$ & 279 \\
16 & $0.00677838[20]$ & 0.00282332 & $248[2]$ & 355 \\
17 & $0.00577854[20]$ & 0.00223324 & $319[2]$ & 448 \\
18 & $0.00518111[20]$ & 0.00177663 & $408[2]$ & 563 \\
19 & $0.00380311[20]$ & 0.00141992 & $521[2]$ & 705 \\
20 & $0.00318213[20]$ & 0.00113876 & $662[2]$ & 879 \\
21 & $0.00267706[20]$ & 0.00091531 & $839[2]$ & 1093 \\
22 & $0.00190205[20]$ & 0.00073636 & $1060[2]$ & 1359 \\
23 & $0.00132755[20]$ & 0.00059204 & $1336[2]$ & 1690 \\
24 & $0.00107286[20]$ & 0.00047489 & $1679[2]$ & 2106 \\
\hline & & & & \\
\hline
\end{tabular}

Table 3.1. Upper bounds for $m_{1}\left(\mathbb{R}^{n}\right)$ and lower bounds for $\chi_{\mathrm{m}}\left(\mathbb{R}^{n}\right)$.

\subsection{Numerical calculations}

A few technical remarks concerning the numerical calculations are in order. For solving the linear programs we use the software lpsolve [3] and we generate the input using the program GP / PARI [16]. We discretize the conditions of the form

$$
z_{0}+z_{1} \Omega_{n}(t)+z_{c}(n+1) \Omega_{n}(t \sqrt{1 / 2-1 /(2 n+2)}) \geq 0 \text { for all } t \geq 0
$$

by discretizing the interval $[0,20]$ into steps of size 0.0005 .

Now we demonstrate in the case $n=4$ how we turn the numerical calculations into a rigorous mathematical proof. The linear program has the optimal numerical solution $z_{0}=0.0826818, z_{1}=0.7660402, z_{c}=0.0302556$. A lower bound of the minimum of the function

$$
z(t)=z_{0}+z_{1} \Omega_{4}(t)+5 z_{c} \Omega_{4}(\sqrt{2 / 5} t)
$$


in $t \in[0,20]$ is -0.00000006 . The function $z(t)$ is positive for $t \geq 20$ because there we have $\Omega_{4}(t) \geq-0.02$ and $\Omega_{4}(\sqrt{2 / 5} t) \geq-0.04$. Thus by adding 0.00000006 to $z_{0}$ we make sure that the new function $z(t)$ is nonnegative. This only slightly affects the value of the bound.

\section{Planar sets avoiding two distances}

In this section we quickly report on the problem of finding the smallest extreme density a measurable set in the plane can have which avoids exactly two distances, i.e., $\inf \left\{m_{d_{1}, d_{2}}\left(\mathbb{R}^{2}\right): d_{1}, d_{2}>0\right\}$. Székely [18] showed that this number is at most $2 / 11$ by giving an upper bound for $m_{1, \sqrt{3}}\left(\mathbb{R}^{2}\right)$. By solving the corresponding linear program on the computer we improve his bound to $m_{1, \sqrt{3}}\left(\mathbb{R}^{2}\right) \leq 0.170213$. By adjusting the distances we can improve this further: $m_{1, j_{1,2} / j_{1,1}}\left(\mathbb{R}^{2}\right) \leq 0.141577$ where $j_{1,1}$ and $j_{1,2}$ are the first two positive zeros of the Bessel function $J_{1}$.

By combining Bukh's result, which we explained in the introduction, with our new bound on $m_{1}\left(\mathbb{R}^{2}\right)$ from the previous section we can improve on this even further:

$$
\inf \left\{m_{d_{1}, d_{2}}\left(\mathbb{R}^{2}\right): d_{1}, d_{2}>0\right\} \leq\left(m_{1}\left(\mathbb{R}^{2}\right)\right)^{2} \leq 0.072046 .
$$

\section{Sets avoiding many distances}

In this section we give a proof of a variant of Bukh's result [5, Theorem 1] about densities of sets avoiding many distances. His proof is based on a so-called zooming out lemma which resembles Szemerédi's regularity lemma for dense graphs, whereas our proof is an easy consequence of Theorem 1.1 and simple properties of the function $\Omega_{n}$.

Theorem 5.1. For every positive integer $N$ there is a number $r=r(N)$ strictly greater than 1 such that for distances $d_{1}, \ldots, d_{N}$ with

$$
d_{2} / d_{1}>r, \quad d_{3} / d_{2}>r, \ldots, d_{N} / d_{N-1}>r
$$

we have $m_{d_{1}, \ldots, d_{N}}\left(\mathbb{R}^{n}\right) \leq 2^{-N}$.

In the proof of Theorem 5.1 some facts about the function $\Omega_{n}$ will be useful. First, we have

$$
\left|J_{0}(t)\right| \leq 1, \quad \text { and } \quad\left|J_{\alpha}(t)\right| \leq 1 / \sqrt{2} \text { for all } \alpha>0 \text { and } t \geq 0
$$

(cf. (4.9.13) in Andrews, Askey, and Roy [1]). From this, it follows at once that $\lim _{t \rightarrow \infty} \Omega_{n}(t)=0$ for $n>2$. For $n=2$ the same follows, e.g., from the asymptotic expansion for $J_{\alpha}$ (cf. (4.8.5) in Andrews, Askey, and Roy [1]).

Moreover,

$$
\Omega_{n}(t) \geq-1 / 2 \quad \text { for all } n \geq 2 \text { and } t \geq 0 \text {. }
$$


To see this, set $\alpha=(n-2) / 2$. It is known that for $\alpha>-1 / 2$ we have

$$
J_{\alpha-1}(t)+J_{\alpha+1}(t)=\frac{2 \alpha}{t} J_{\alpha}(t)
$$

(cf. Andrews, Askey, and Roy [1, (4.6.5)]). Combining this identity with (1) we obtain

$$
\begin{aligned}
\Omega_{n}(t) & =\Gamma(\alpha+1)\left(\frac{2}{t}\right)^{\alpha} \frac{t}{2 \alpha}\left(J_{\alpha-1}(t)+J_{\alpha+1}(t)\right) \\
& =\Omega_{n-2}(t)+\Gamma(\alpha)\left(\frac{2}{t}\right)^{\alpha-1} J_{\alpha+1}(t) .
\end{aligned}
$$

Now, recall that the global minimum of $\Omega_{n}$ is attained at $j_{\alpha+1,1}$, the first positive zero of $J_{\alpha+1}$ (cf. Section 3). From (14), we have $\Omega_{n}\left(j_{\alpha+1,1}\right)=\Omega_{n-2}\left(j_{\alpha+1,1}\right)$. It follows that the minimum of $\Omega_{n}$ is at least the minimum of $\Omega_{n-2}$. To finish the proof of (13) we have to check $\Omega_{2}$ and $\Omega_{3}$, which can be easily accomplished.

Proof of Theorem 5.1. Given $N>0$, set $\varepsilon=1 /\left(N 2^{N+1}\right)$. Since $\Omega_{n}(0)=1$ and since $\Omega_{n}$ is continuous, there is a number $t_{0}>0$ such that $\Omega_{n}(t)>1-\varepsilon$ for $t \leq t_{0}$. Likewise, since $\lim _{t \rightarrow \infty} \Omega_{n}(t)=0$, there is a number $t_{1}>t_{0}$ such that $\left|\Omega_{n}(t)\right|<\varepsilon$ for $t \geq t_{1}$.

Set $r=r(N)=t_{1} / t_{0}$ and let distances $d_{1}, \ldots, d_{N}$ be given such that (11) is satisfied. We claim that, for $1 \leq j \leq N$,

$$
\sum_{i=j}^{N} \frac{1}{2^{N-i+1}} \cdot \Omega_{n}\left(t d_{i}\right) \geq-\frac{1}{2^{N-j+2}}-(N-j) \varepsilon .
$$

Before we prove the claim, we show how to apply it. By taking $j=1$ in the claim, and since by our choice of $\varepsilon$ we have $-(N-1) \varepsilon \geq-1 / 2^{N+1}$, it follows that

$$
\sum_{i=1}^{N} \frac{1}{2^{N-i+1}} \cdot \Omega_{n}\left(t d_{i}\right) \geq-\frac{1}{2^{N}} .
$$

Now we may set $z_{0}=1 / 2^{N}$ and $z_{i}=1 / 2^{N-i+1}$ for $i=1, \ldots, N$ and apply Theorem 1.1 . proving our result.

To finish, we prove the claim by induction. For $j=N$, the statement follows immediately from (13). Now, suppose the statement is true for some $1<j \leq N$. We show that it is also true for $j-1$ by distinguishing two cases.

First, for $t \leq t_{0} / d_{j-1}$, we see from the choice of $t_{0}$ that $\Omega_{n}\left(t d_{j-1}\right)>1-\varepsilon$. Using this and the induction hypothesis, we then obtain

$$
\begin{aligned}
\sum_{i=j-1}^{N} \frac{1}{2^{N-i+1}} \cdot \Omega_{n}\left(t d_{i}\right) & =\frac{1}{2^{N-j+2}} \cdot \Omega_{n}\left(t d_{j-1}\right)+\sum_{i=j}^{N} \frac{1}{2^{N-i+1}} \cdot \Omega_{n}\left(t d_{i}\right) \\
& \geq \frac{1-\varepsilon}{2^{N-j+2}}-\frac{1}{2^{N-j+2}}-(N-j) \varepsilon \\
& \geq-\frac{1}{2^{N-j+3}}-(N-j+1) \varepsilon
\end{aligned}
$$


Now suppose $t \geq t_{0} / d_{j-1}$. Observe that, for $j \leq i \leq N$, we have $t d_{i} \geq t_{0} d_{i} / d_{j-1} \geq$ $t_{0} r=t_{1}$, hence $\left|\Omega_{n}\left(t d_{i}\right)\right|<\varepsilon$. So, by using [13], we have

$$
\begin{aligned}
\sum_{i=j-1}^{N} \frac{1}{2^{N-i+1}} \cdot \Omega_{n}\left(t d_{i}\right) & =\frac{1}{2^{N-j+2}} \cdot \Omega_{n}\left(t d_{j-1}\right)+\sum_{i=j}^{N} \frac{1}{2^{N-i+1}} \cdot \Omega_{n}\left(t d_{i}\right) \\
& \geq-\frac{1}{2^{N-j+3}}-(N-j+1) \varepsilon,
\end{aligned}
$$

finishing the proof of the claim.

Acknowledgments. We thank Christine Bachoc for helpful discussions, Noam D. Elkies for enlightening comments, and Boris Bukh and Lex Schrijver for their valuable suggestions on our manuscript.

The first author was partially supported by CAPES/Brazil under grant BEX 2421/04-6. The second author was partially supported by the Deutsche Forschungsgemeinschaft (DFG) under grant SCHU 1503/4. The second author thanks the Hausdorff Research Institute for Mathematics (Bonn) for its hospitality and support.

\section{References}

[1] Andrews, G. E., Askey, R., Roy, R.: Special Functions. Cambridge Univ. Press (1999) Zbl 0920.33001 MR 1688958

[2] Bachoc, C., Nebe, G., de Oliveira Filho, F. M., Vallentin, F.: Lower bounds for measurable chromatic numbers. Geom. Funct. Anal. 19, 645-661 (2009) Zbl pre05652303 MR 2563765

[3] Berkelaar, M., Eikland, K., Notebaert, P.: lpsolve version 5.5. Available from the web site fhttp://lpsolve.sourceforge.net/

[4] Bourgain, J.: A Szemerédi type theorem for sets of positive density in $\mathbb{R}^{k}$. Israel J. Math. 54, 307-316 (1986) Zbl 0609.10043 MR 0853455

[5] Bukh, B.: Measurable sets with excluded distances. Geom. Funct. Anal. 18, 668-697 (2008) Zbl 1169.52005 MR 2438995

[6] Cohn, H., Elkies, N. D.: New upper bounds on sphere packings I. Ann. of Math. 157, 689-714 (2003) Zbl 1041.52011 MR 1973059

[7] Croft, H. T.: Incidence incidents. Eureka (Cambridge) 30, 22-26 (1967)

[8] Croft, H. T., Falconer, K. J., Guy, R. K.: Unsolved Problems in Geometry. Springer (1991) ZBL 0748.52001 MR 1107516

[9] Delsarte, P.: An algebraic approach to the association schemes of coding theory. Philips Res. Rep. Suppl. 10, vi+97 pp. (1973) Zbl 1075.05606 MR 0384310

[10] Falconer, K. J.: The realization of distances in measurable subsets covering $\mathbb{R}^{n}$. J. Combin. Theory Ser. A 31, 184-189 (1981) Zbl 0469.05021 MR 0629593

[11] Falconer, K. J.: The realization of small distances in plane sets of positive measure. Bull. London Math. Soc. 18, 475-477 (1986) Zbl 0599.28009 MR 0847987

[12] Falconer, K. J., Marstrand, J. M.: Plane sets with positive density at infinity contain all large distances. Bull. London Math. Soc. 18, 471-474 (1986) Zbl 0599.28008 MR 0847986

[13] Furstenberg, H., Katznelson, Y., Weiss, B.: Ergodic theory and configurations in sets of positive density. In: Mathematics of Ramsey Theory, J. Nešetřil and V. Rödl (eds.), Springer, 184-198 (1990) Zbl 0738.28013 MR 1083601 
[14] Katznelson, Y.: An Introduction to Harmonic Analysis. Dover Publ. (1976) Zbl 0352.43001 MR 0422992

[15] Mattila, P.: Geometry of Sets and Measures in Euclidean Spaces: Fractals and Rectifiability. Cambridge Univ. Press (1995) Zbl 0819.28004 MR 1333890

[16] PARI-group: PARI/GP version 2.3.3. Bordeaux (2006); http://pari.math.u-bordeaux.fr

[17] Schoenberg, I. J.: Metric spaces and completely monotone functions. Ann. of Math. 39, 811841 (1938) Zbl 0019.41503 MR 1503439

[18] Székely, L. A.: Measurable chromatic number of geometric graphs and sets without some distances in Euclidean space. Combinatorica 4, 213-218 (1984) Zbl 0558.05020 MR 0771730

[19] Székely, L. A.: Erdős on unit distances and the Szemerédi-Trotter theorems. In: Paul Erdôs and his Mathematics, G. Halász et al. (eds.), Springer, 649-666 (2002) Zbl 1035.05037 MR 1954746

[20] Székely, L. A., Wormald, N. C.: Bounds on the measurable chromatic number of $\mathbb{R}^{n}$. Discrete Math. 75, 343-372 (1989) Zbl 0683.05021 MR 1001407

[21] Watson, G. N.: A Treatise on the Theory of Bessel Functions. Cambridge Univ. Press (1995) Zbl 0849.33001 MR 1349110 